\title{
A Biomimetic Combination of Actives Enhances Skin Hydration and Barrier Function via Modulation of Gene Expression: Results of Two Double-Blind, Vehicle-Controlled Clinical Studies
}

\author{
Silke Altgilbers ${ }^{a} \quad$ Frank Rippke $^{a} \quad$ Alexander Filbry $^{a} \quad$ Stefanie Conzelmann ${ }^{a}$ \\ Jens-Peter Vietzke ${ }^{a}$ Thorsten Burkhardt ${ }^{a}$ Dörte Segger ${ }^{b}$ \\ Dennis Roggenkamp ${ }^{a}$ Elke Grönniger ${ }^{a}$

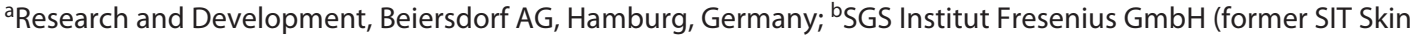 \\ Investigation and Technology), Hamburg, Germany
}

\section{Keywords}

Xerosis cutis · Dry skin · Gene expression · Urea · Natural moisturizing factor $\cdot$ Ceramide

\begin{abstract}
Introduction: Xerosis cutis is characterized by a decreased stratum corneum (SC) hydration and an impaired skin barrier function. Urea, the most prevalent natural moisturizing factor (NMF), is currently considered the gold standard. Its efficacy can further be increased by combining urea with other NMF and skin barrier lipids (SBLs). Objective: We set out to evaluate physiological effects of a novel functional moisturizer containing 10\% urea, additional NMF components, and a combination of SBLs on skin hydration and skin barrier integrity on a cellular and phenotypic level in female volunteers suffering from xerosis. Methods: Two doubleblind, vehicle-controlled clinical studies were conducted. In the first study, 44 female subjects having very dry body skin applied the moisturizer or its vehicle twice daily to their volar forearms. Twenty-four hours after a single product application as well as $24 \mathrm{~h}$ after 2 weeks of treatment, SC hydration was measured by corneometry. Skin barrier function was assessed by transepidermal water loss $24 \mathrm{~h}$ and $48 \mathrm{~h}$ after 2
\end{abstract}

karger@karger.com www.karger.com/spp

Karger $\frac{1}{\%}$
(C) 2021 The Author(s)

Published by S. Karger AG, Basel

This is an Open Access article licensed under the Creative Commons Attribution-NonCommercial-4.0 International License (CC BY-NC) (http://www.karger.com/Services/OpenAccessLicense), applicable to the online version of the article only. Usage and distribution for commercial purposes requires written permission. weeks of regular use. Twenty-four hours after 2 weeks of application, skin tape stripping was performed, and urea content was determined in the 3rd strip by means of high-performance liquid chromatography/tandem mass spectrometry. In the second study, 22 women with self-reported very dry skin applied the moisturizer or vehicle twice daily to their volar forearms for 2 weeks. Then, suction blister samples were obtained for gene expression analysis using RT-PCR. Results: Application of the actives led to significantly improved skin hydration and barrier function at all points in time. Compared to the vehicle, application of the moisturizer for 2 weeks resulted in a significant increase in SC urea content. Relative gene expression data revealed significant upregulation of genes associated with skin barrier function, hydration, differentiation, and lipid metabolism compared to the vehicle-treated area. Conclusions: Overall, our data demonstrate that the functional moisturizer provides an adequate bioavailability of urea and a beneficial biophysical impact on xerotic skin. Topical treatment with a combination of urea and additional NMF as well as SBL can modify mRNA expression of important epidermal genes stimulating cellular processes and functions. The well-tolerated novel

Dennis Roggenkamp and Elke Grönniger contributed equally.
Correspondence to:

Elke Grönniger, elke.groenniger@ beiersdorf.com 
functional moisturizer stimulates molecular mechanisms involved in skin hydration and barrier function and is a profoundly effective treatment option for xerosis cutis.

(C) 2021 The Author(s)

Published by S. Karger AG, Basel

\section{Introduction}

Xerosis cutis is a common skin condition. Many patients experience dry, scaly, and rough skin often associated with reduced elasticity and wrinkling. Further, erythema as well as fissures or rhagades, especially of the feet, can develop [1]. Dry skin may feel irritated and tight and is also prone to pruritus. Especially, this pruritus can lead to a considerable impairment in patients' quality of life [2, $3]$. The predominantly affected parts are skin areas with a reduced density of sebaceous glands such as the outer aspects of lower legs, forearms, hands, and feet.

Xerosis cutis is characterized by a decreased water content and an impaired barrier function of the stratum corneum (SC). In many cases of dry skin, a lack of natural moisturizing factor (NMF) and skin barrier lipids (SBLs) has been shown in the SC. NMF consists of a complex, hydroscopic composition mainly comprising free amino acids, pyrrolidone carboxylic acid (PCA), lactates, and urea. SBLs, which constitute a hydrophobic domain in the profound layer of the SC, are predominantly composed of ceramides (40-50\%), cholesterol (25\%), and free fatty acids (10-15\%) [1, 4-7].

For the treatment of dry skin conditions, moisturizing formulas containing humectants are essential. Urea, which has been used for more than a century in the management of dermatological disorders, is currently considered the gold standard in the treatment of xerosis cutis [1, 6]. As an element of the NMF, urea represents an important hygroscopic component and thus contributes to the maintenance of skin hydration [8]. Urea improves the skin barrier function as well as cutaneous defense and hydration mechanisms. It enhances penetration of active ingredients and exhibits antipruritic as well as keratolytic effects [1].

Urea therapy has been associated with few adverse effects and is generally well tolerated by xerotic skin. Both the safety and efficacy of urea have been largely established over the past hundred years [6-10].

The mode of action of urea, though, has not yet been fully understood. Due to its hygroscopic properties, urea has long been considered as "just" a humectant. However, more recent studies demonstrate that urea is also actively involved in epidermal gene regulation impacting kerati- nocyte differentiation, lipid synthesis, AMP production, epidermal permeability, and barrier function $[6,11,12]$. We hypothesized that not only urea, as reported by Grether-Beck and others $[6,11,12]$, but also its combination with other NMF components and SBLs can influence epidermal gene expression, thus impacting dry-skin physiology beyond a physical mode of action. According to this hypothesis, we sought to determine the effect of a novel functional moisturizer containing a biomimetic combination of $10 \%$ urea and additional NMF components (lactate, amino acids, and PCA) as well as SBLs (ceramide NP, cholesterol, and linoleic acid-rich sunflower seed oil), further referred to as the "Urea plus NMF \& SBL Complex," on the expression pattern of diverse skin- and barrier-relevant genes. Furthermore, we hypothesized that the formulation containing this complex shows significantly higher efficacy in terms of moisturization and skin barrier repair than that of the vehicle formulation.

In our studies, we took an integrated approach, investigating effects of the functional moisturizer in female volunteers with dry skin on (i) the ex vivo penetration profile of urea, (ii) the ex vivo expression of genes encoding for proteins involved in skin barrier function, hydration, and lipid metabolism, and (iii) in vivo skin barrier and hydration parameters in subjects suffering from dry to very dry skin.

\section{Materials and Methods}

\section{Study Formulations}

The ingredients of the functional moisturizer containing the "Urea plus NMF \& SBL Complex" (Eucerin 10\% Urea Foot Cream, Beiersdorf AG, Hamburg, Germany) and the vehicle are presented in Table 1.

Study I

The first single-center, randomized, double-blind, vehiclecontrolled study was conducted at SIT Skin Investigation and Technology Hamburg GmbH, Hamburg, Germany. In this study, 44 healthy, female subjects (aged 22-65 years) were enrolled. The major inclusion criteria were female gender and a self-reported very dry body skin. Finally, only volunteers with objectively dry to very dry skin were included (corneometer units below 40). Patients with skin diseases or dermatological disorders were excluded from the study. During a 1-week washout preconditioning period, eligible subjects were required to use a special mild skin cleansing product (Doppeldusch Fresh, Beiersdorf AG, Hamburg, Germany) twice daily on their inner forearms for approximately $60 \mathrm{~s}$. During this preconditioning period and throughout the whole study, volunteers had to refrain from using other skin-care products as well as special skin-cleansing products such as bath or shower oils on the arms (inside and outside). Also, intensive UV exposure (solarium or sun) was prohibited. 
Table 1. Ingredients of the functional moisturizer containing the "Urea plus NMF \& SBL Complex" and its vehicle

\begin{tabular}{lll}
\hline INCl & Moisturizer & Vehicle \\
\hline Lactic acid + aqua & $\mathrm{X}$ & \\
Urea & $\mathrm{X}$ & \\
Cholesterol + tocopherol & $\mathrm{X}$ & \\
Carnitine & $\mathrm{X}$ & \\
Aqua + sodium lactate & $\mathrm{X}$ & \\
Ceramide NP & $\mathrm{X}$ & \\
Arginine HCL & $\mathrm{X}$ & \\
Alanine & $\mathrm{X}$ & \\
Glycine & $\mathrm{X}$ & \\
Aqua + sodium PCA & $\mathrm{X}$ & \\
Helianthus annuus seed oil & $\mathrm{X}$ & \\
Sodium chloride & $\mathrm{X}$ & \\
Glycerin & $\mathrm{X}$ & $\mathrm{X}$ \\
Dimethicone & $\mathrm{X}$ & $\mathrm{X}$ \\
Octyldodecanol & $\mathrm{X}$ & $\mathrm{X}$ \\
Hydrogenated coco-glycerides & $\mathrm{X}$ & $\mathrm{X}$ \\
Ethylhexyl cocoate & $\mathrm{X}$ & $\mathrm{X}$ \\
Caprylic/capric triglyceride & $\mathrm{X}$ & $\mathrm{X}$ \\
Sorbitan stearate + aqua & $\mathrm{X}$ & $\mathrm{X}$ \\
Glyceryl stearate SE & $\mathrm{X}$ & $\mathrm{X}$ \\
Glyceryl stearate & $\mathrm{X}$ & $\mathrm{X}$ \\
Sodium cetearyl sulfate & $\mathrm{X}$ & $\mathrm{X}$ \\
Distarch phosphate & $\mathrm{X}$ & $\mathrm{X}$ \\
Phenoxyethanol & $\mathrm{X}$ & $\mathrm{X}$ \\
Cetearyl alcohol & $\mathrm{X}$ & $\mathrm{X}$ \\
Carbomer & $\mathrm{X}$ & $\mathrm{X}$ \\
Aqua & & \\
\hline
\end{tabular}

NMF, natural moisturizing factor; SBL, skin barrier lipid; $P C A$, pyrrolidone carboxylic acid.

On the first study day, test areas were established on the inner forearms for treatment with the verum or the vehicle and baseline measurements were performed. There were 4 test sites, namely 2 on each inner forearm. Three of the sites were treated with the test products, and one site was left untreated and served as a control. Positioning of treatment locations within the test sites was permutated from subject to subject by means of a randomization scheme.

To assess skin hydration, a Corneometer ${ }^{\circledR}$ CM 825 (MDD 4 device, Courage+Khazaka, Cologne, Germany) was utilized according to the EEMCO guidelines [13]. The device had been validated in a multicentric ring study [14].

Ten measurements were taken per test site, and the median result was reported as arbitrary units. For transepidermal water loss (TEWL) measurements, the DermaLab ${ }^{\circledR}$ (Cortex, Hadsund, Denmark) was used in accordance with the EEMCO guidance [13]. The probe had been validated in earlier randomized, double-blind, vehicle-controlled trials with similar moisturizers, yielding TEWL data paralleled by clinical grading scores of visible dryness and tactile roughness [15]. Moreover, another double-blind study comparing wound-healing ointments confirmed the association of changes in TEWL with investor gradings of dioxide laser wounds [16].
Then, verum and the vehicle were once applied to their allocated test sites by a technician. Each formulation was dispensed from a disposable syringe in an amount equaling $2 \mu \mathrm{L} / \mathrm{cm}^{2}$. The technician then carefully spread the formula using a finger, protected by a finger cot. On the uncovered test sites, formulations could absorb for at least $5 \mathrm{~min}$. Measurements were performed by trained and experienced personnel after acclimatization for at least 30 min under standard atmospheric conditions $\left(21.5^{\circ} \mathrm{C} \pm 1.0^{\circ} \mathrm{C}\right.$ and $50 \% \pm 5 \%$ relative humidity).

Twenty-four hours after the controlled application of the test formulations, skin hydration of the test areas was assessed. Further, subjects were supplied with one unit of each test formulation (lasting for 2 weeks) for self-application at home, written usage instructions, and a diary for recording test material application times. The investigator trained subjects in correct self-application for regular use. After 1 week of test formula use at home, a compliance check for all volunteers including an examination of all diaries for correct product application was performed at the institute. Additionally, subjects had to demonstrate the application of the test formulation under supervision of the investigator.

After 2 weeks of regular use ( $24 \mathrm{~h}$ after the last application), skin hydration, and TEWL were assessed again. Furthermore, test sites were stripped with adhesive tape using D-Squame ${ }^{\circledR}$ (DSQ) Standard Sampling Discs (22 mm diameter; CuDerm Corp., Dallas, TX, USA) as described by Knott et al. [17]. Only slight decreases in urea content with the number of tape strips were reported after topical application of $10 \%$ urea solution for 3-6 h [18]. However, as the first 2 strips may be contaminated with the product, these were discarded, and the 3rd DSQ per test site was used for stratified urea analyses. The SC protein content on each DSQ was analyzed using the SquameScan ${ }^{\mathrm{TM}} 850 \mathrm{~A}$ device (Heiland electronic $\mathrm{GmbH}$, Wetzlar, Germany) by measuring the absorption (\%) indicating the protein content (see online suppl. Table 1; for all online suppl. material, seewww.karger.com/doi/10.1159/000520009). After measurements, DSQs were rolled up (sample side inwards), placed in provided tubes on dry ice, and stored at $-80^{\circ} \mathrm{C}$ until further use. Twenty-four hours later (48 $\mathrm{h}$ after the last application), a final TEWL assessment was performed.

\section{Urea Analytics}

We extracted the DSQs with $1 \mathrm{~mL}$ of methanol/water $50 / 50$ (v:v). The extract was then filtered using a $0.2-\mu \mathrm{m}$ PTFE membrane filter (Pall Corporation Acrodisc $13 \mathrm{~mm}$ minispike with 0.2 $\mu \mathrm{m}$ PTFE).

The analytical quantitative determination of urea was performed by means of high-performance liquid chromatography/ tandem mass spectrometry using an Agilent HPLC 1200 series (Agilent Technologies GmbH, Waldbronn, Germany) in combination with a 3200 QTRAP (AB Sciex, Darmstadt, Germany). Parameters for high-performance liquid chromatography were as follows: column: Hamilton HPLC anion exchange PRP-100 $125 \times$ $2 \mathrm{~mm}, 10 \mu \mathrm{m}$ (Sigma-Aldrich, Taufkirchen, Germany) and mobile phase (gradient): solvent A: water with ammonia containing 2 $\mathrm{mmol} / \mathrm{L}$ ammonium carbonate $(\mathrm{pH} 10)$ and solvent $\mathrm{B}$ : acetonitrile. The flow rate was $400 \mu \mathrm{L} / \mathrm{min}$, and the injection volume $2 \mu \mathrm{L}$. Samples were applied to the column at $60 \% \mathrm{~B}(0 \mathrm{~min})$ and eluted with $20 \%$ solvent B (5 and $12 \mathrm{~min}$ ) and then $60 \%$ solvent B (13 and 18 min). The retention time of urea was $1.5 \mathrm{~min}$.

Electrospray detection was carried out by means of "multireaction monitoring" of the transition of the precursor-ion to a prod- 
uct-ion (precursor-ion $[\mathrm{m} / \mathrm{z}]=61.0$; product-ion $[\mathrm{m} / \mathrm{z}]=44.1$; positive polarity). Instead of application of an internal standard, quantitative determination was performed by means of external standard calibration (Urea, Sigma-Aldrich 99.5\%, lot \#SZBF0850V) for quantification (linear regression; coefficient of determination $\left.R^{2}=0.998\right)$. The limit of determination was $0.3 \mu \mathrm{g}$ urea, and the signal-to-noise ratio was $>10: 1$. Blank runs showed no interfering signal of relevant size. Multiple analyses showed a relative standard deviation of $8 \%$ (day-to-day precision).

We integrated all values into our analysis, whereas all measurements below the detection limit of 0.1 were set as 0 . Results were depicted relative to the absorption (\%) of the corresponding DSQ, measured via SquameScan ${ }^{\mathrm{TM}}$ and displayed in $\mu \mathrm{g} / \mathrm{DSQ}$. Hence, the data were normalized for variations in protein content.

\section{Study II}

The second single-center, randomized, double-blind, vehiclecontrolled study was conducted at SIT Skin Investigation and Technology Hamburg GmbH, Hamburg, Germany. Twenty-two healthy, female subjects (aged 25-65 years) with self-reported very dry body skin and corneometer units below 40 a.u. at the test sites were included into this single-center, randomized, double-blind, vehicle-controlled home-in-use study to evaluate the effects of the novel moisturizer containing the "Urea plus NMF \& SBL Complex" on gene expression levels in human skin ex vivo. Patients with chronic or acute skin disease (e.g., atopic dermatitis [AD] and psoriasis) at the skin test site(s) were excluded from the study. During a 2 -week preconditioning period and throughout the whole study, volunteers had to refrain from using topical preparations for antibacterial, anti-allergic, or immunosuppressive treatment of the skin at the test site.

On the first study day, test areas were established on the volar forearms of each volunteer for treatment with verum or the vehicle. Volunteers applied the test formulations twice daily for 14 days according to written instructions. After 2 weeks, 2 suction blisters were generated in each test area, and suction blister epidermis was isolated as previously described $[19,20]$ (see online suppl. Table 2).

\section{Gene Expression Analysis of Suction Blister Samples}

Each suction blister roof was collected in a separate cryo vial containing RNAlater ${ }^{\mathrm{TM}}$ stabilization solution (Thermo Fisher Scientific, Waltham, USA) and stored at $4^{\circ} \mathrm{C}$ overnight. For isolation of total RNA, suction blister roofs were disrupted and homogenized using the Precellys ${ }^{\circledR} 24$ tissue homogenizer (Bertin Technologies, Montigny-le-Bretonneux, France). Cell debris was removed by centrifugation (Eppendorf 5417R Refrigerated Centrifuge, Eppendorf, Hamburg, Germany). Clear supernatants were used to isolate total RNA utilizing the RNeasy ${ }^{\circledR}$ Mini Kit (Qiagen, Hilden, Germany) according to the manufacturer's protocol. After reverse transcription using the High-Capacity cDNA Reverse Transcription Kit (Applied Biosystems, Foster City, CA, USA), mRNA levels of genes associated with skin barrier function, hydration, and lipid metabolism were detected using the Real-Time TaqMan ${ }^{\circledR}$ PCR (Applied Biosystems). Quantification was achieved using the $2^{-\Delta \Delta \mathrm{Ct}}$ method, calculating the relative gene expression changes of the target genes in verum-treated versus vehicle-treated samples. The Ct values of both (verum and the vehicle) were normalized to the endogenous housekeeping gene GAPDH $(\Delta \mathrm{Ct})$ : $\Delta \Delta \mathrm{Ct}=\Delta \mathrm{Ct}($ verum $)-\Delta \mathrm{Ct}$ (vehicle).

NMF and Lipids Modulate Gene

Expression in Xerosis

\section{Statistics}

Statistical Analysis

Statistical analyses were performed using Microsoft Excel 2010 and the Microsoft Excel 2013 (XP; Microsoft Corp., Redmond, WA, USA), the SAS Software Package for Windows V9.4 (SAS Institute $\mathrm{GmbH}$, Heidelberg, Germany), and STATISTICA 12.0 (StatSoft, Inc., Tulsa, OK, USA). All statistical tests were two-sided at significance level alpha $=0.05$.

For study I, the less pronounced effect could be expected for TEWL measurements (c.f. delta $=0.75, \mathrm{SD}=1.73$ ). To achieve a power of at least $80 \%$ with a paired $t$-test, a sample of $n=44$ was necessary. For corneometry, a higher effect was expected, thus $n=$ 44 was considered sufficient here.

For study II, based on our experience with gene expression analyses in suction blisters of subjects with dry skin in a similar study design [21], we derived empirically the minimum number of subjects needed. Significance (adjusted $p$ value $\leq 0.05$; experimentwise error rate is controlled) is tested by applying Wilcoxon's signed rank test with $p$ value adjustment according to Benjamini and Hochberg; in order to control, the false discovery rate was used.

Skin Moisturization and Skin Barrier Function

Data were tested for normality using Shapiro-Wilk's test. If applicable, post hoc pairwise comparisons were assessed using the Fisher LSD test.

Urea Content in DSQs and Relative Gene Expression Data of Suction Blister Samples

Comparison between the untreated test site, verum, and the vehicle was performed using Wilcoxon's signed-rank test. Kendall rank correlation tests were performed to detect correlations between urea content in DSQs and TEWL.

\section{Results}

\section{Tolerability}

Results showed that all test formulations were well tolerated. Neither an incompatibility reaction nor discomfort was observed or reported for any subject. All subjects completed the studies.

\section{The Novel Moisturizer Improves Skin Hydration and \\ Barrier Integrity}

\section{Skin Hydration}

The average pretreatment baseline corneometer mean value of $24.6 \pm 5.6$ a.u. on the test sites (inner forearm) indicated the very dry skin condition of the volunteers which did not significantly change during the treatment period as the corneometer values of the untreated control sites show (after 24 h: $2.70 \pm 3.46$ a.u., after 1 week: 6.97 \pm 5.27 a.u.). As illustrated in Figure 1a, in comparison to the vehicle control $(7.10 \pm 3.46$ a.u.), sites treated with verum (10.27 \pm 4.49 a.u.) showed a statistically significant 


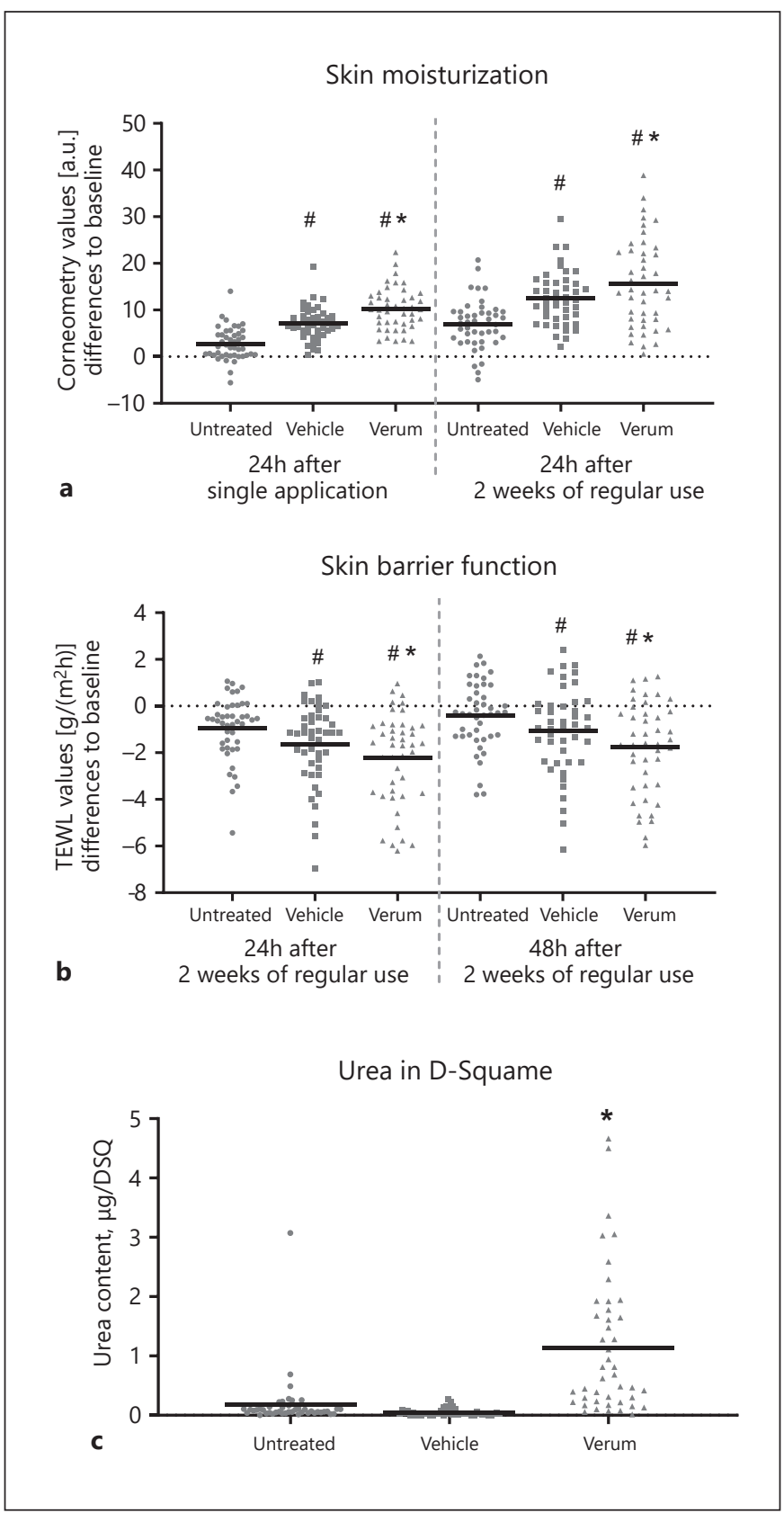

Fig. 1. NMF and skin-related lipids enhance skin hydration and barrier function in a vehicle-controlled treatment of xerosis. a Skin moisturization was measured by corneometry $24 \mathrm{~h}$ after a single product application as well as $24 \mathrm{~h}$ after 2 weeks of regular use $(n=44)$. b Assessment of TEWL was conducted $24 \mathrm{~h}$ and $48 \mathrm{~h}$ after 2 weeks of regular use $(n=44)$. c Urea content in DSQs collected $24 \mathrm{~h}$ after 2 weeks of regular use $(n=44)$. Results are shown in a scatter plot as individual values, and the line indicates the mean. Significant differences are marked with an asterisk (* for $p<0.05$ comparison to vehicle; ${ }^{\#}$ for $p<0.05$ comparison to baseline). NMF, natural moisturizing factor; TEWL, transepidermal water loss; DSQ, D-Squame. increase in corneometry values $24 \mathrm{~h}$ after a single and controlled application $(p<0.0001)$. Twenty-four hours after the 2-week treatment period, compared to the vehicle control (12.48 \pm 5.74 a.u.), corneometry values of verum-treated sites were significantly increased to 15.67 \pm 9.55 a.u. $(p=0.0012)$.

\section{Skin Barrier Function}

As the pretreatment baseline level for TEWL, a mean value of $7.19 \pm 2.2\left(\mathrm{~g} / \mathrm{m}^{2} \mathrm{~h}\right)$ was detected on all test sites of the included volunteers. The skin barrier condition of the untreated control sites did not significantly change during the treatment period as shown by the TEWL values (after $24 \mathrm{~h}:-0.94 \pm 1.34 \mathrm{~g} /\left[\mathrm{m}^{2} \mathrm{~h}\right]$ and after 1 week: -0.42 $\left.\pm 1.43 \mathrm{~g} /\left[\mathrm{m}^{2} \mathrm{~h}\right]\right)$. In comparison to vehicle-control sites $\left(-1.64 \pm 1.74 \mathrm{~g} /\left[\mathrm{m}^{2} \mathrm{~h}\right]\right)$, TEWL values of verum-treated areas demonstrated a significant decrease $(-2.23 \pm 1.96 \mathrm{~g} /$ $\left.\left[\mathrm{m}^{2} \mathrm{~h}\right]\right) 24 \mathrm{~h}$ after the 2 -week treatment period $(p=0.0021)$. Forty eight hours after 14 days of application, verumtreated areas $\left(-1.78 \pm 1.98 \mathrm{~g} /\left[\mathrm{m}^{2} \mathrm{~h}\right]\right)$ demonstrated significantly lower TEWL values than the vehicle-treated sites $\left(-1.07 \pm 1.87 \mathrm{~g} /\left[\mathrm{m}^{2} \mathrm{~h}\right]\right), p=0.0004$, Fig. $\left.1 \mathrm{~b}\right)$.

\section{Urea Penetrates into the Skin}

Figure 1c depicts the urea content in DSQ $24 \mathrm{~h}$ after the 2 -week treatment period. The average value of urea content detected in the untreated test sites was $0.18 \pm 0.46$ $\mu \mathrm{g} / \mathrm{DSQ}$. For the vehicle-treated areas, a urea content of $0.05 \pm 0.06 \mu \mathrm{g} / \mathrm{DSQ}$ was determined, while sites treated with verum displayed a urea content of $1.14 \pm 1.19 \mu \mathrm{g} /$ DSQ. Compared to the vehicle-treated site, the urea content in the verum-treated area was significantly increased $(p<0.0001)$. After verum treatment, a highly significant negative correlation $(-0.53 ; p<0.001)$ was detected between DSQ urea content and TEWL.

\section{The Novel Moisturizer Containing the "Urea plus NMF \& SBL Complex" Stimulates Cellular Processes to Maintain Cellular Functions}

First, as shown in Figure 2, relative gene expression data of suction blister roofs from volunteers having applied the novel moisturizer containing the "Urea plus NMF \& SBL Complex" for 2 weeks revealed, compared to the vehicle, significant upregulation (adjusted $p$ value $<0.05)$ of the following genes involved in skin differentiation and barrier function: transglutaminase 1 (TGM1), involucrin $(I V L)$, loricrin $(L O R)$, filaggrin $(F L G)$, filaggrin family member 2 (FLG2), keratin-10 (KRT10), kallikrein 5 (SC tryptic enzyme, KLK5), kallikrein 7 (SC chymotryptic enzyme, KLK7), caspase-14 (CASP14), cor- 
Fig. 2. NMF and skin-related lipids enhance expression of genes involved in skin differentiation and barrier function, hydration, and lipid metabolism. Significant regulation of gene expression (adjusted $p<$ 0.05) detected in verum-treated suction blister samples compared to the vehicle $(n=22)$. Results are depicted as mean. Significant differences are marked with an asterisk (* for $p<0.05$ comparison to vehicle), a fold change $>1.5$ is marked with \#. NMF, natural moisturizing factor.

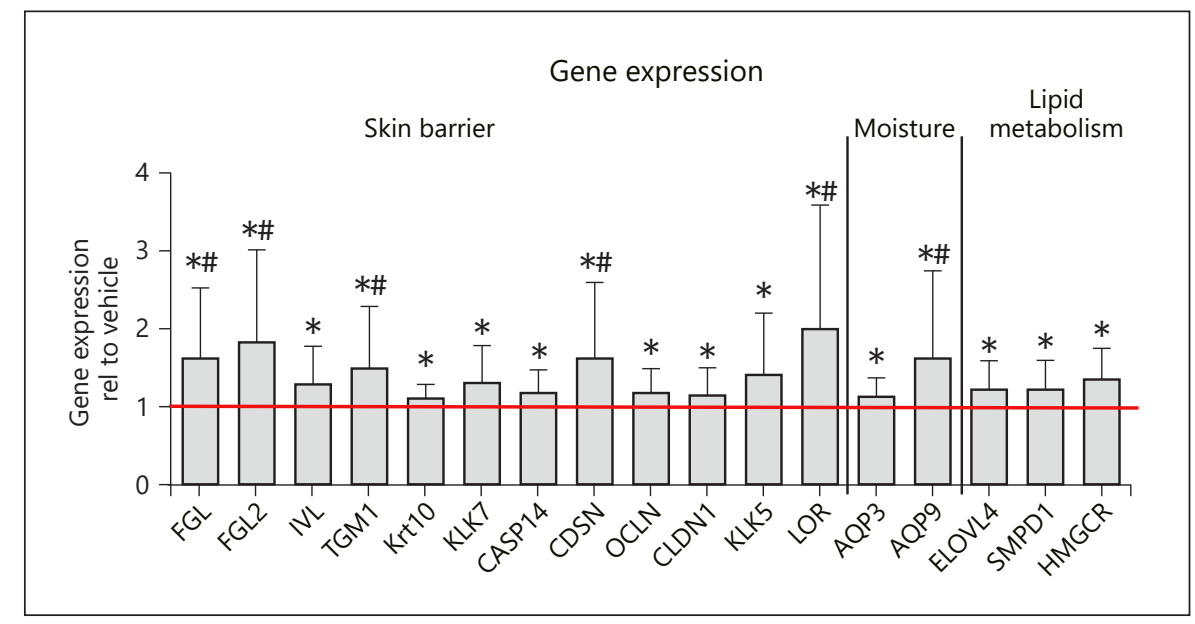

neodesmosin (CDSN), occludin (OCLN), and claudin-1 (CLDN1). Second, expression of genes associated with hydration such as aquaporin (AQP)-3 (AQP3) and aquaporin-9 (AQP9) was significantly increased compared to the vehicle. Third, the gene expression levels of markers for skin lipid metabolism such as elongation of very longchain fatty acids-4 (ELOVL4), sphingomyelin phosphodiesterase (SMPD1), and 3-hydroxy-3-methylglutaryl coenzyme A reductase (HMGCR) were, compared to the vehicle, significantly upregulated.

\section{Discussion}

The prevalence of dry skin in the German adult population was demonstrated to be almost $30 \%$ of the population older than 16 years [22]. A much higher proportion of xerosis up to $100 \%$ was found in geriatric communities in Germany and Thailand [23, 24]. These numbers emphasize the need for a sufficient disease management [22]. An internationally recognized approach in the treatment of xerosis cutis is the application of moisturizers containing lipophilic and hydrophilic active substances. Hydrophilic compounds include components of the NMF such as urea, amino acids, and PCA. Lipophilic substances related to SBLs include ceramides, cholesterol, and natural oils rich in linoleic acid $[1,4,5]$.

Here, we determined the efficacy and molecular mechanisms of a novel moisturizer containing a biomimetic combination of $10 \%$ urea and additional NMF components (lactate, amino acids, and PCA) as well as SBLs (ceramide NP, cholesterol, and linoleic acid-rich sunflower seed oil) referred to as the "Urea plus NMF \&
SBL Complex" in female subjects suffering from dry to very dry skin. First, we investigated changes in skin hydration after treatment with the new formulation compared to the vehicle. As early as $24 \mathrm{~h}$ after a single application of the test formulation, corneometry values were significantly increased compared to vehicle-treated areas. This difference was further expanded $24 \mathrm{~h}$ after 2 weeks of treatment, indicating that application of the moisturizer improves skin hydration fast and incrementally. These findings were corroborated by our urea bioavailability data and in accordance with recent tapestripping studies observing significant higher concentration of urea in the deeper SC layers compared to other small solutes [25].

Compared to vehicle treatment, the application of the novel moisturizer with the "Urea plus NMF \& SBL Complex" led to significantly reduced TEWL values $24 \mathrm{~h}$ and $48 \mathrm{~h}$ after the 2-week treatment period. Moreover, the highly significant negative correlation between urea content in DSQs and TEWL underlines the positive effect of urea on skin barrier function. This outcome is in line with earlier studies. Urea has been shown to reduce TEWL [26-29] and improve skin hydration [28-34] in various dry-skin conditions. With respect to hydrating effects, a combination of urea with NMF and SBL was superior to treatment with vehicle alone or topical formulations only containing urea $[1,34]$. As with our study, also vehicletreated sites demonstrated a significant improvement of skin hydration and skin barrier function. Accordingly, a recent review concluded that emollient vehicles with moisturizing effects are therapeutically advantageous on multiple levels [35]. 
Beyond its humectant effects on the SC, urea belongs to the group of osmolytes, small water-soluble polar molecules with low vapor pressure involved in water homeostasis in the skin [36]. In case of epidermal osmotic stress resulting from dry environmental conditions or skin diseases, urea can replace water and thereby stabilize cell membranes to prevent dehydration-induced structural reorganizations and phase transitions of lipid bilayer systems $[37,38]$.

Treatment of the SC with urea at reduced relative humidity results in molecular and macroscopic SC responses comparable to those observed by an increase in relative humidity without urea application [39]. Moreover, recent genomic and transcriptomic analyses disclosed that urea modulates the expression of multiple genes involved in epidermal lipid and antimicrobial peptide synthesis, urea transport proteins, keratinocyte differentiation, and desmolysis. Hence, urea profoundly impacts epidermal structure and function as a regulator of hydration, barrier formation, and desquamation $[6,11,12]$.

The first step to elucidate the mode of action of the novel functional moisturizer with the "Urea plus NMF \& SBL Complex" in more detail was to investigate the bioavailability of urea. By determining the ex vivo penetration profile of urea in DSQs from volunteers after application of the moisturizer for 2 weeks, we confirmed that urea was effectively released from the formulation to the skin.

Next, we elucidated the effects of the "Urea plus NMF \& SBL Complex" on the expression of genes encoding for proteins involved in skin hydration, differentiation, lipid metabolism, and barrier function. Although the observed fold changes were rather small, the analysis revealed significant changes for multiple genes associated with specific cellular functions with respect to the comparison verum versus vehicle. All depicted genes showed a significant expression change $(p<0.05)$; differently expressed genes with a foldchange $>1.5$ were highlighted [40].

First, we investigated genes involved in skin differentiation and barrier function: Specifically, FLG, FLG2, IVL, $T G M 1$, and $L O R$ play a crucial role in epidermal differentiation [41]. Filaggrin represents a skin barrier protein with an important function in keratin filament alignment [42]. Also, its degradation products yield NMF [43], and FLG gene mutations have been associated with an increased risk for AD and ichthyosis vulgaris [44, 45]. Furthermore, elevating FLG protein levels by $5-10 \%$ may provide clinical benefits in the management of patients with dry skin and atopy $[44,46]$. Our results are in line with earlier studies reporting that TGM1, IVL, LOR, and FLG mRNA and protein levels were slightly but significantly increased after $10 \%$ urea application compared to the untreated skin [12]. The effects of the "Urea plus NMF \& SBL Complex" were observed although both study formulations contained glycerin which also upregulates mRNA level expression of FLG and $L O R$ in keratinocytes [47]. However, Hoppe et al. [48] did not find any major changes in gene activity, including $L O R$ and $F L G$, in patients suffering from $\mathrm{AD}$ and/or ichthyosis vulgaris after a 4-week treatment period, with moisturizers containing $20 \%$ glycerin or $5 \%$ urea, although significant reductions in dryness scores and TEWL were observed. We further investigated the expression of CASP14, a crucial protease involved in FLG catabolism and NMF generation [49]. Differentiation-related KRT10, as well as the cornified envelope-associated proteins $I V L$ and $L O R$ were found to be suppressed in human $\mathrm{AD}$ [50]. Additionally, the expression of KRT10 was reduced in dry skin [51] and AD lesions, potentially related to a disturbed barrier function [52]. Moreover, CLDN1 and OCLN are considered relevant to skin barrier function as they are involved in the formation of proteins essential for tight-junction function [53]. In mice, deletion of CLDN1 led to death within $24 \mathrm{~h}$ of birth due to massive TEWL [53].

Application of the "Urea plus NMF \& SBL Complex" resulted in an increase in CASP14, KRT10, IVL, LOR, $C L D N 1$, and OCLN gene expression, which was accompanied by the observed improvement of skin barrier function, exceeding the expression differences of $I V L$ and $L O R$ reported by Grether-Beck et al. [12] after a 10\% urea treatment ex vivo.

Moreover, we observed an induction of gene expression of KLK5, KLK7, and CDSN. Both KLK5 and KLK7 have been demonstrated to cleave $C D S N$ which is crucial in corneocyte cohesion; hence, its degradation is necessary for desquamation [54]. In dry-skin conditions such as winter or atopic xerosis, skin levels of KLK5 and KLK7 are diminished, providing a basis for "corneotherapy" of disturbed desquamation [55]. However, an elevation of KLK5 and KLK7 levels was observed in AD in association with biomarkers also in nonlesional skin, requiring further investigations of epidermal serine protease activity $[55,56]$.

The second functional cluster we investigated represents genes that are associated with epidermal hydration: $A Q P 3$ and $A Q P 9$ are members of the aquaglyceroporin family, membrane proteins that build water channels across the cell membrane enabling transport of water and small solutes such as glycerin and urea [57]. Activation of 
$A Q P$ expression is a prerequisite of sustained epidermal water supply and skin hydration as evidenced by earlier studies demonstrating that mice lacking AQP3 exhibited a reduced glycerol content and a diminished water holding capacity in the epidermis [58]. Also, an AQP3-deficient epidermis displays reduced skin elasticity and delayed wound healing [59]. AQP3 has been linked to keratinocyte proliferation, migration, differentiation, and survival [60]. Here, we show the upregulation of $A Q P$ gene expression ex vivo, which was previously only demonstrated in keratinocytes in vitro [12]. These findings indicate efficacy of the "Urea plus NMF \& SBL Complex" in $A Q P 3$ and $A Q P 9$ upregulation after topical application, improving skin hydration inside-out.

The third group of genes that we focused on were genes involved in lipid production and metabolism: the ELO family of proteins is involved in the elongation of long-chain fatty acids. ELOVL4 mRNA increases during keratinocyte differentiation in vivo and in vitro [61]. ELOVL4 plays a crucial role in the synthesis of $\omega$-Oacylceramides, and depletion of these omega-O-acylceramides has been associated with skin conditions exhibiting a skin barrier dysfunction such as $\mathrm{AD}[62,63]$. Also, studies with mice lacking functional ELOVL4 demonstrate a strong reduction in very-long-chain FFAs and a defective skin water permeability barrier function [63]. $S M P D 1$ encodes for acid sphingomyelinase, converting sphingomyelin to ceramide. In $\mathrm{AD}$, a decreased SMPD1 activity was demonstrated in lesional and nonlesional skin, correlating with reduced SC ceramide content and disturbed barrier function [52]. Cholesterol synthesis is regulated by the enzyme HMG CoA reductase. Following acute barrier disruption, epidermal cholesterol synthesis increases accompanied by an increase in HMGCR activity, protein, and mRNA levels [64]. These earlier findings emphasize the high relevance of the genes we analyzed. Our study revealed a slight but significant upregulation of the mRNA expression of ELOVL4, SMPD1, and HMGCR compared to vehicle-treated sites.

A limitation of the study was the inclusion of female volunteers only. Gender-specific differences in skin response to the investigated complex remain to be clarified in future studies.

Taken together as the outcome, the "Urea plus NMF \& SBL Complex" modifies mRNA expression of important epidermal genes beyond its outside-in humectant properties. The gene expression data provide evidence for the induction of cellular epidermal processes resulting in an improved skin hydration and barrier function inside-out, exceeding a passive humectant effect. As hypothesized, the whole complex revealed significant changes in the gene expression pattern of barrier relevant proteins. The expression differences were detected ex vivo in more genes than already described for a $10 \%$ urea treatment [12]. These results further elucidate the molecular mechanisms stimulated by the moisturizer. Furthermore, our findings advance our understanding why urea has been the ingredient of choice for the treatment of xerosis for many decades. Future studies will have to investigate its potential role in more specific skin conditions such as atopic, diabetic, and senile xerosis.

\section{Acknowledgments}

The authors would like to thank Dr. Silke Gallinat for her support in preparing the manuscript, Ursula Holtzman for supporting the RNA extraction, and Claudia Rauscher and Andre Rehage for the statistical analyses.

\section{Statement of Ethics}

The protocol of study I was approved by an Institutional Review Board of SIT Skin Investigation and Technology Hamburg $\mathrm{GmbH}$, Hamburg, Germany, and the second study was approved by the Independent Ethics Committee Freiburg (feki code 08/2610). For both studies, the recommendations of the current version of the Declaration of Helsinki and the guideline of the International Conference on Harmonization Good Clinical Practice (ICH GCP) were observed as applicable to a nondrug study. All volunteers provided written, informed consent.

\section{Conflict of Interest Statement}

Silke Altgilbers, Frank Rippke, Alexander Filbry, Stefanie Conzelmann, Jens-Peter Vietzke, Thorsten Burkhardt, Dennis Roggenkamp, and Elke Grönniger are employees of Beiersdorf AG. Dörte Segger is an employee of SGS Institut Fresenius GmbH (former SIT Skin Investigation and Technology), Hamburg, Germany. None of the authors state a conflict of interest.

\section{Funding Sources}

The study was sponsored by Beiersdorf AG, Hamburg, Germany.

\section{Author Contributions}

S.A., F.R., A.F., S.C., J.-P.V., T.B., D.S., D.R., E.G. D.R. and E.G. conceived and designed experiments. S.A., D.S. T.B., and J.D. performed the experiments. S.A., D.S., T.B., J.-P.V., D.R., 
E.G., and D.S. analyzed data. A.F. contributed reagents/materials/analysis tools. F.R., D.R., and E.G. wrote the paper. A.F., S.C., and J.-P.V. assisted in study conception, design, and interpretation of results.

\section{References}

1 Augustin M, Wilsmann-Theis D, Körber A, Kerscher M, Itschert G, Dippel M, et al. Diagnosis and treatment of xerosis cutis - a position paper. J Dtsch Dermatol Ges. 2019; 17(Suppl 7):3-33.

2 Garibyan L, Chiou AS, Elmariah SB. Advanced aging skin and itch: addressing an unmet need. Dermatol Ther. 2013;26(2):92-103.

3 Ständer S, Augustin M, Reich A, Blome C, Ebata T, Phan NQ, et al. International Forum for the Study of Itch Special Interest Group scoring itch in clinical trials. Pruritus assessment in clinical trials: consensus recommendations from the International Forum for the Study of Itch (IFSI) Special Interest Group scoring itch in clinical trials. Acta Derm Venereol. 2013;93:509-14.

4 Del Rosso JQ, Levin J. The clinical relevance of maintaining the functional integrity of the stratum corneum in both healthy and diseaseaffected skin. J Clin Aesthet Dermatol. 2011; 4(9):22-42.

5 Fowler J. Understanding the role of natural moisturizing factor in skin hydration. Pract Dermatol. 2012;7:36-40.

6 Friedman AJ, von Grote EC, Meckfessel MH. Urea: a clinically oriented overview from bench to bedside. J Drugs Dermatol. 2016; 15(5):633-39.

7 Celleno L. Topical urea in skincare: a review. Dermatol Ther. 2018;31(6):e12690.

8 Berardesca E, Cameli N. Non-invasive assessment of urea efficacy: a review. Int J Clin Pract. 2020;74(S187):e13603.

9 Alan Andersen F. Final report of the safety of urea. Int J Toxicol. 2004;24(Suppl 3):1-56.

10 Pan M, Heinecke G, Bernardo S, Tsui C, Levitt J. Urea: a comprehensive review of the clinical literature. Dermatol Online J. 2013; 19(11):20392.

11 Buraczewska I, Berne B, Lindberg M, Lodén $M$, Törmä $H$. Long-term treatment with moisturizers affects the mRNA levels of genes involved in keratinocyte differentiation and desquamation. Arch Dermatol Res. 2009; 301(2):175-81.

12 Grether-Beck S, Felsner I, Brenden H, Kohne Z, Majora M, Marini A, et al. Urea uptake enhances barrier function and antimicrobial defense in humans by regulating epidermal gene expression. J Invest Dermatol. 2012;132(6): 1561-72.

13 Berardesca E, Loden M, Serup J, Masson P, Rodrigues LM. The revised EEMCO guidance for the in vivo measurement of water in the skin. Skin Res Technol. 2018;24:351-8.

\section{Data Availability Statement}

Data are made available upon reasonable request to the author.
14 Heinrich U, Koop U, Leneveu-Duchemin MC, Osterrieder K, Bielfeldt S, Chkarnat C, et al. Multicentre comparison of skin hydration in terms of physical-, physiological- and product-dependent parameters by the capacitive method (corneometer CM 825). Int J Cosmet Sci. 2003;25(1-2):45-53.

15 Weber TM, Kausch M, Rippke F, Schoelermann AM, Filbry AW. Treatment of xerosis with a topical formulation containing glyceryl glucoside, natural moisturizing factors, and ceramide. J Clin Aesthet Dermatol. 2012;5(8): 29-39.

16 Trookman NS, Rizer RL, Weber T. Treatment of minor wounds from dermatologic procedures: a comparison of three topical wound care ointments using a laser wound model. J Am Acad Dermatol. 2011;64(3 Suppl):S8-15.

17 Knott A, Achterberg V, Smuda C, Mielke H, Sperling G, Dunckelmann K, et al. Topical treatment with coenzyme Q10-containing formulas improves skin's Q10 level and provides antioxidative effects. Biofactors. 2015; 41(6):383-90.

18 Lodén M, Boström P, Kneczke M. Distribution and keratolytic effect of salicylic acid and urea in human skin. Skin Pharmacol Physiol. 1995;8(4):173-8.

19 Kiistala U. Suction blister device for separation of viable epidermis from dermis. J Invest Dermatol. 1968;50:129-37.

20 Südel KM, Venzke K, Knußmann-Hartig E, Moll I, Stäb F, Wenck H, et al. Tight control of matrix metalloproteinase- 1 activity in human skin. Photochem Photobiol. 2003;78: 355-60.

21 Schrader A, Siefken W, Kueper T, Breitenbach U, Gatermann C, Sperling G, et al. Effects of glyceryl glucoside on AQP3 expression, barrier function and hydration of human skin. Skin Pharmacol Physiol. 2012; 25(4):192-9.

22 Augustin M, Kirsten N, Körber A, WilsmannTheis D, Itschert G, Staubach-Renz P, et al. Prevalence, predictors and comorbidity of dry skin in the general population. J Eur Acad Dermatol Venereol. 2019;33(1):147-50.

23 Hahnel E, Blume-Peytavi U, Trojahn C, Dobos G, Jahnke I, Kanti V, et al. Prevalence and associated factors of skin diseases in aged nursing home residents: a multicentre prevalence study. BMJ Open. 2017;7(9):e018283.

24 Rojvutthikun I, Nitayavardhana S. Descriptive cross-sectional survey of xerosis in general middle-aged and elderly at Benchakiti Park hospital. RSU International Research Conference; 2020. p. 369-79.
25 Intarakumhaeng R, Alsheddi L, Wanasathop A, Shi Z, Li SK. Skin permeation of urea under finite dose condition. J Pharm Sci. 2019;108: 987-95.

26 Loden M. Urea-containing moisturizers influence barrier properties of normal skin. Arch Dermatol Res. 1996;288(2):103-7.

27 Loden M. Barrier recovery and influence of irritant stimuli in skin treated with a moisturizing cream. Contact Dermatitis. 1997;36(5): 256-60.

28 Borelli C, Bielfeldt S, Borelli S, Schaller M, Korting HC. Cream or foam in pedal skin care: towards the ideal vehicle for urea used against dry skin. Int J Cosmet Sci. 2001;33(1): $37-43$.

29 Pan M, Heinecke G, Bernardo S, Tsui C, Levitt J. Urea: a comprehensive review of the clinical literature. Dermatol Online J. 2013; 19(11):20392.

30 Schölermann A, Banke-Bochita J, Bohnsack K, Rippke F, Herrmann WM. Efficacy and safety of Eucerin ${ }^{\circledR} 10 \%$ urea lotion in the treatment of symptoms of aged skin. J Dermatol Treat. 1998;9:175-9.

31 Schölermann A, Bohnsack K, Stephan K, Banke-Bochita J, Herrmann WM, Rippke F. Wirksamkeit und verträglichkeit von Eucerin salbe $10 \%$ urea bei xerotischer AltershautErgebnisse einer Vehikel-kontrollierten klinischen Doppelblindstudie. Z Hautkr. 1999;10(74):557-62.

32 Bohnsack K, Tausch I, Gassmüller J, Rippke F. Wirksamkeit auf das Symptom trockene Haut und Langzeitverträglichkeit von Laceran ${ }^{\circledR}$ Lotion $10 \%$ urea bei Patienten mit atopischem Ekzem. Z Hautkr. 1997;1(72):349.

33 Wilhelm KP, Schölermann A, Bohnsack K, Wilhelm D, Rippke F. Wirksamkeit und Verträglichkeit einer topischen Zubereitung mit $10 \%$ Urea (Laceran Salbe 10\% Urea) bei Neurodermitis. Akt Dermatol. 1998;24:26-30.

34 Weber TM, Kausch M, Rippke F, Schoelermann AM, Filbry AW. Treatment of xerosis with a topical formulation containing glyceryl glucoside, natural moisturizing factors, and ceramide. J Clin Aesthet Dermatol. 2012;5(8): 29-39.

35 Danby SG, Draelos ZD, Stein Gold LF, Cha A, Vlahos B, Aikman L, et al. Vehicles for atopic dermatitis therapies: more than just a placebo. J Dermatolog Treat. 2020:1-14. [published online ahead of print, $2020 \mathrm{Jul} 16]$.

36 El-Chami C, Haslam IS, Steward MC, O’Neill CA. Role of organic osmolytes in water homoeostasis in skin. Exp Dermatol. 2014;23(8): $534-7$. 
37 Costa-Balogh FO, Wennerström H, Wadsö L, Sparr E. How small polar molecules protect membrane systems against osmotic stress: the urea-water-phospholipid system. J Phys Chem B. 2006;110(47):23845-52.

38 Pham QD, Wolde-Kidan A, Gupta A, Schlaich A, Schneck E, Netz RR, et al. Effects of urea and TMAO on lipid self-assembly under osmotic stress conditions. J Phys Chem B. 2018; 122(25):6471-82.

39 Mojumdar EH, Pham QD, Topgaard D, Sparr E. Skin hydration: interplay between molecular dynamics, structure and water uptake in the stratum corneum. Sci Rep. 2017;7(1) 15712.

40 McCarthy DJ, Smyth GK. Testing significance relative to a fold-change threshold is a TREAT. Bioinformatics. 2009;25(6):765-71.

41 Chen CS, Lavker RM, Rodeck U, Risse B, Jensen PJ. Use of a serum-free epidermal culture model to show deleterious effects of epidermal growth factor on morphogenesis and differentiation. J Invest Dermatol. 1995;104(1): $107-12$.

42 Brown SJ, Irvine AD. Atopic eczema and the filaggrin story. Semin Cutan Med Surg. 2008; 27(2):128-37.

43 Mao-Qiang M, Fowler AJ, Schmuth M, Lau P, Chang S, Brown BE, et al. Peroxisome-proliferator-activated receptor (PPAR)-gamma activation stimulates keratinocyte differentiation. J Invest Dermatol. 2004;123(2):305-12.

44 Brown SJ, Kroboth K, Sandilands A, Campbell LE, Pohler E, Kezic S, et al. Intragenic copy number variation within filaggrin contributes to the risk of atopic dermatitis with a dose-dependent effect. J Invest Dermatol. 2012;132(1):98-104.

45 Kezic S, Kemperman PM, Koster ES, de Jongh CM, Thio HB, Campbell LE, et al. Loss-offunction mutations in the filaggrin gene lead to reduced level of natural moisturizing factor in the stratum corneum. J Invest Dermatol. 2008;128(8):2117-9.

$46 \mathrm{McGrath}$ JA. Profilaggrin, dry skin, and atopic dermatitis risk: size matters. J Invest Dermatol. 2012;132(1):10-11.
47 Páyer E, Szabó-Papp J, Ambrus L, Szöllősi AG, Andrási M, Dikstein S, et al. Beyond the physico-chemical barrier: glycerol and xylitol markedly yet differentially alter gene expression profiles and modify signalling pathways in human epidermal keratinocytes. Exp Dermatol. 2018;27(3):280-4.

48 Hoppe T, Winge MC, Bradley M, Nordenskjöld M, Vahlquist A, Törmä H, et al. Moisturizing treatment of patients with atopic dermatitis and ichthyosis vulgaris improves dry skin, but has a modest effect on gene expression regardless of FLG genotype. J Eur Acad Dermatol Venereol. 2015;29(1):174-7.

49 Hoste E, Kemperman P, Devos M, Denecker G, Kezic S, Yau N, et al. Caspase-14 is required for filaggrin degradation to natural moisturizing factors in the skin. J Invest Dermatol. 2011;131(11):2233-41.

50 Bao L, Mohan GC, Alexander JB, Doo C, Shen $\mathrm{K}$, Bao J, et al. A molecular mechanism for IL-4 suppression of loricrin transcription in epidermal keratinocytes: implication for atopic dermatitis pathogenesis. Innate Immun. 2017;23(8):641-7.

51 Engelke M, Jensen JM, Ekanayake-Mudiyanselage S, Proksch E. Effects of xerosis and ageing on epidermal proliferation and differentiation. Br J Dermatol. 1997;137(2):219-25.

52 Jensen JM, Fölster-Holst R, Baranowsky A, Schunck M, Winoto-Morbach S, Neumann C, et al. Impaired sphingomyelinase activity and epidermal differentiation in atopic dermatitis. I Invest Dermatol. 2004;122(6):1423-31.

53 Furuse M, Hata M, Furuse K, Yoshida Y, Haratake A, Sugitani Y, et al. Claudin-based tight junctions are crucial for the mammalian epidermal barrier: a lesson from claudin-1-deficient mice. J Cell Biol. 2002;156(6):1099-111.

54 Caubet C, Jonca N, Brattsand M, Guerrin M, Bernard D, Schmidt R, et al. Degradation of corneodesmosome proteins by two serine proteases of the kallikrein family, SCTE/ KLK5/hK5 and SCCE/KLK7/hK7. I Invest Dermatol. 2004;122(5):1235-44.
55 Rawlings AV, Voegeli R. Stratum corneum proteases and dry skin conditions. Cell Tissue Res. 2013;351(2):217-35.

56 Nomura H, Suganuma M, Takeichi T, Kono M, Isokane Y, Sunagawa K, et al. Multifaceted analyses of epidermal serine protease activity in patients with atopic dermatitis. Int $\mathrm{J} \mathrm{Mol}$ Sci. 2020;21:913.

57 Tardelli M, Stulnig TM. Aquaporin regulation in metabolic organs. Vitam Horm. 2020; 112:71-93.

58 Ma T, Hara M, Sougrat R, Verbavatz JM, Verkman AS. Impaired stratum corneum hydration in mice lacking epidermal water channel aquaporin-3. J Biol Chem. 2002; 277(19):17147-53.

59 Hara M, Ma T, Verkman AS. Selectively reduced glycerol in skin of aquaporin-3-deficient mice may account for impaired skin hydration, elasticity, and barrier recovery. J Biol Chem. 2002;277(48):46616-21.

60 Bollag WB, Aitkens L, White J, Hyndman KA Aquaporin-3 in the epidermis: more than skin deep. Am J Physiol Cell Physiol. 2020 318(6):C1144-53.

61 Mizutani Y, Sun H, Ohno Y, Sassa T, Wakashima T, Obara M, et al. Cooperative synthesis of ultra long-chain fatty acid and ceramide during keratinocyte differentiation. PLoS One. 2013;8(6):e67317.

62 Vasireddy V, Uchida Y, Salem N Jr, Kim SY, Mandal NA, Reddy GB, et al. Loss of functional ELOVL4 depletes very long-chain fatty acids $(>$ or $=\mathrm{C} 28)$ and the unique omega-Oacylceramides in skin leading to neonatal death. Hum Mol Genet. 2007;16(5):471-82.

63 Cameron DJ, Tong Z, Yang Z, Kaminoh J, Kamiyah S, Chen H, et al. Essential role of Elovl4 in very long chain fatty acid synthesis, skin permeability barrier function, and neonatal survival. Int J Biol Sci. 2007;3(2):111-9.

64 Feingold KR. Thematic review series: skin lipids. The role of epidermal lipids in cutaneous permeability barrier homeostasis. J Lipid Res. 2007;48(12):2531-46. 